IJMMS 27:12 (2001) 715-723

PII. S0161171201011097

http://ijmms.hindawi.com

(c) Hindawi Publishing Corp.

\title{
ON THE SPECTRUM OF THE DISTRIBUTIONAL KERNEL RELATED TO THE RESIDUE
}

\author{
AMNUAY KANANTHAI
}

(Received 22 June 2000 and in revised form 26 February 2001)

\begin{abstract}
We study the spectrum of the distributional kernel $K_{\alpha, \beta}(x)$, where $\alpha$ and $\beta$ are complex numbers and $x$ is a point in the space $\mathbb{R}^{n}$ of the $n$-dimensional Euclidean space. We found that for any nonzero point $\xi$ that belongs to such a spectrum, there exists the residue of the Fourier transform $(-1)^{k} \widehat{K_{2 k, 2 k}(\xi)}$, where $\alpha=\beta=2 k, k$ is a nonnegative integer and $\xi \in \mathbb{R}^{n}$.
\end{abstract}

2000 Mathematics Subject Classification. 46F10, 46F12.

1. Introduction. Gel'fand and Shilov [2, pages 253-256] have studied the generalized function $P^{\lambda}$, where

$$
P=\sum_{i=1}^{p} x_{i}^{2}-\sum_{j=p+1}^{p+q} x_{j}^{2}
$$

is a quadratic form, $\lambda$ is a complex number, and $p+q=n$ is the dimension of $\mathbb{R}^{n}$. They found that $P^{\lambda}$ has two sets of singularities, namely $\lambda=-1,-2, \ldots,-k, \ldots$ and $\lambda=-n / 2,-n / 2-1, \ldots,-n / 2-k, \ldots$, where $k$ is a positive integer. For the singular point $\lambda=-k$, the generalized function $P^{\lambda}$ has a simple pole with residue

$$
\frac{(-1)^{k}}{(k-1) !} \delta_{1}^{(k-1)}(P) \quad \text { or } \quad \operatorname{res}_{\lambda=-k} P^{\lambda}=\frac{(-1)^{k}}{(k-1) !} \delta_{1}^{(k-1)}(P)
$$

for $p+q=n$ is odd with $p$ odd and $q$ even. Also, for the singular point $\lambda=-n / 2-k$ they obtained

$$
\operatorname{res}_{\lambda=-n / 2-k} P^{\lambda}=\frac{(-1)^{q / 2} L^{k} \delta(x)}{2^{2 k} k ! \Gamma((n / 2)+k)}
$$

for $p+q=n$ is odd with $p$ odd and $q$ even.

Now, let $K_{\alpha, \beta}(x)$ be the convolution of the functions $R_{\alpha}^{H}(u)$ and $R_{\beta}^{\ell}(v)$, that is,

$$
K_{\alpha, \beta}(x)=R_{\alpha}^{H}(u) * R_{\beta}^{\ell}(v),
$$

where $R_{\alpha}^{H}(u)$ and $R_{\beta}^{\ell}(v)$ are defined by (2.1) and (2.3), respectively. Since $R_{\alpha}^{H}(u)$ and $R_{\beta}^{\ell}(v)$ are tempered distributions, see [4, pages 30-31], thus $K_{\alpha, \beta}(x)$ is also a tempered distribution and is called the distributional kernel.

In this paper, we use the idea of Gel'fand and Shilov to find the residue of the Fourier transform $(-1)^{k} \widehat{K_{2 k, 2 k}(\xi)}$, where $K_{2 k, 2 k}$ is defined by (1.4) with $\alpha=\beta=2 k$ and $k$ is a nonnegative integer. We found that for any nonzero point $\xi$ that belongs to the spectrum of $(-1)^{k} K_{2 k, 2 k}(x)$, there exists the residue of the Fourier transform 
$(-1)^{k} \widehat{K_{2 k, 2 k}(\xi)}$. Actually $(-1)^{k} K_{2 k, 2 k}(x)$ is an elementary solution of the operator $\diamond^{k}$ iterated $k$ times, that is, $\diamond^{k}\left[(-1)^{k} K_{2 k, 2 k}(x)\right]=\delta$, where $\delta$ is the Dirac-delta distribution.

The operator $\diamond^{k}$ was first introduced by Kananthai [4] and named as the Diamond operator defined by

$$
\diamond^{k}=\left[\left(\frac{\partial^{2}}{\partial x_{1}^{2}}+\frac{\partial^{2}}{\partial x_{2}^{2}}+\cdots+\frac{\partial^{2}}{\partial x_{p}^{2}}\right)^{2}-\left(\frac{\partial^{2}}{\partial x_{p+1}^{2}}+\frac{\partial^{2}}{\partial x_{p+2}^{2}}+\cdots+\frac{\partial^{2}}{\partial x_{p+q}^{2}}\right)^{2}\right]^{k},
$$

where $p+q=n$ is the dimension of $\mathbb{R}^{n}$.

Moreover, the operator $\diamond^{k}$ can be expressed as the product of the operators $\square^{k}$ and $\triangle^{k}$, that is,

$$
\diamond^{k}=\square^{k} \triangle^{k}=\triangle^{k} \square^{k}
$$

where $\square^{k}$ is an ultra-hyperbolic operator iterated $k$ times defined by

$$
\square^{k}=\left(\sum_{i=1}^{p} \frac{\partial^{2}}{\partial x_{i}^{2}}-\sum_{j=p+1}^{p+q} \frac{\partial^{2}}{\partial x_{j}^{2}}\right)^{k},
$$

where $p+q=n$. The operator $\triangle^{k}$ is an elliptic operator or Laplacian iterated $k$ times defined by

$$
\triangle^{k}=\left(\frac{\partial^{2}}{\partial x_{1}^{2}}+\frac{\partial^{2}}{\partial x_{2}^{2}}+\cdots+\frac{\partial^{2}}{\partial x_{n}^{2}}\right)^{k}
$$

Trione [7, page 11] has shown that the function $R_{2 k}^{H}(u)$ defined by (2.1) with $\alpha=2 k$ is an elementary solution of the operator $\square^{k}$. Also, Aguirre Téllez [1, pages 147-148] has proved that the solution $R_{2 k}^{H}(u)$ exists only for odd $n$ with $p$ odd and $q$ even $(p+q=n)$. Moreover, we can show that the function $(-1)^{k} R_{2 k}^{\ell}(v)$ is an elementary solution of the operator $\triangle^{k}$, where $R_{2 k}^{\ell}(v)$ is defined by (2.3) with $\beta=2 k$.

\section{Preliminaries}

DEFINITION 2.1. Let $x=\left(x_{1}, x_{2}, \ldots, x_{n}\right)$ be a point of $\mathbb{R}^{n}$, and write $u=x_{1}^{2}+$ $x_{2}^{2}+\cdots+x_{p}^{2}-x_{p+1}^{2}-\cdots-x_{p+q}^{2}, p+q=n$. Denote by $\Gamma_{+}=\left\{x \in \mathbb{R}^{n}: x_{1}>0, u>0\right\}$ the set of an interior of the forward cone, and $\overline{\Gamma_{+}}$denotes the closure of $\Gamma_{+}$. For any complex number $\alpha$, define

$$
R_{\alpha}^{H}(u)= \begin{cases}\frac{u^{(\alpha-n) / 2}}{K_{n}(\alpha)}, & \text { for } x \in \Gamma_{+}, \\ 0, & \text { for } x \notin \Gamma_{+},\end{cases}
$$

where the constant $K_{n}(\alpha)$ is given by the formula

$$
K_{n}(\alpha)=\frac{\pi^{(n-1) / 2} \Gamma((2+\alpha-n) / 2) \Gamma((1-\alpha) / 2) \Gamma(\alpha)}{\Gamma((2+\alpha-p) / 2) \Gamma((p-\alpha) / 2)} .
$$

The function $R_{\alpha}^{H}(u)$ is called the ultra-hyperbolic kernel of Marcel Riesz and was introduced by Nozaki [6, page 72]. The function $R_{\alpha}^{H}$ is an ordinary function or classical function if $\operatorname{Re}(\alpha) \geq n$ and is a distribution of $\alpha$ if $\operatorname{Re}(\alpha)<n$. Let $\operatorname{supp} R_{\alpha}^{H}(u) \subset \overline{\Gamma_{+}}$, where $\operatorname{supp} R_{\alpha}^{H}(u)$ denotes the support of $R_{\alpha}^{H}(u)$. 
DEFINITION 2.2. Let $x=\left(x_{1}, x_{2}, \ldots, x_{n}\right)$ be a point of $\mathbb{R}^{n}$, and write $v=x_{1}^{2}+$ $x_{2}^{2}+\cdots+x_{n}^{2}$. For any complex number $\beta$, define

$$
R_{\beta}^{\ell}(v)=\frac{2^{-\beta} \pi^{-n / 2} \Gamma((n-\beta) / 2) v^{(\beta-n) / 2}}{\Gamma(\beta / 2)} .
$$

The function $R_{\beta}^{\ell}(v)$ is called the elliptic kernel of Marcel Riesz and is an ordinary function for $\operatorname{Re}(\beta) \geq n$ and is a distribution of $\beta$ for $\operatorname{Re}(\beta)<n$.

DEFINITION 2.3. Let $f$ be a continuous function, then the Fourier transform of $f$, denoted by $\mathfrak{I} f$ or $\hat{f}(\xi)$, is defined by

$$
\mathfrak{J} f=\hat{f}(\xi)=\frac{1}{(2 \pi)^{n / 2}} \int_{\mathbb{R}^{n}} e^{-i(\xi, x)} f(x) d x,
$$

where $x=\left(x_{1}, x_{2}, \ldots, x_{n}\right) \in \mathbb{R}^{n}, \xi=\left(\xi_{1}, \xi_{2}, \ldots, \xi_{n}\right) \in \mathbb{R}^{n}$, and $(\xi, x)=\xi_{1} x_{1}+\xi_{2} x_{2}+$ $\cdots+\xi_{n} x_{n}$. From (2.4), the inverse Fourier transform of $\hat{f}(\xi)$ is defined by

$$
f(x)=\mathfrak{J}^{-1} \hat{f}(\xi)=\frac{1}{(2 \pi)^{n / 2}} \int_{\mathbb{R}^{n}} e^{i(\xi, x)} \hat{f}(\xi) d x .
$$

If $f$ is a distribution with compact support, by [8, Theorem 7.4.3, page 187] (2.5) can be written as

$$
\mathfrak{J} f=\hat{f}(\xi)=\frac{1}{(2 \pi)^{n / 2}}\left\langle f(x), e^{-i(\xi, x)}\right\rangle .
$$

LEMMA 2.4. Given the equation

$$
\diamond^{k} u(x)=\delta
$$

where $\diamond^{k}$ is the operator defined by (1.5), and $\delta$ is the Dirac-delta distribution, $u(x)$ is an unknown, $k$ is a nonnegative integer and $x \in \mathbb{R}^{n}$, where $n$ is odd with $p$ odd, q even $(n=p+q)$. Then $u(x)=(-1)^{k} K_{2 k, 2 k}(x)$ is an elementary solution of the operator $\diamond^{k}$. Here $K_{2 k, 2 k}(x)=R_{2 k}^{H}(u) * R_{2 k}^{\ell}(v)$ from (1.4) with $\alpha=\beta=2 k$.

Proof. See [4, page 33].

In this paper, we study the spectrum of $(-1)^{k} K_{2 k, 2 k}(x)$, relate to the residue of the Fourier transform $(-1)^{k} \widehat{K_{2 k, 2 k}(\xi)}$.

LEMMA 2.5. The Fourier transform

$$
\begin{aligned}
\widehat{K_{\alpha, \beta}(\xi)}= & (2 \pi)^{n / 2} \mathfrak{J} R_{\alpha}^{H}(u) \mathfrak{J} R_{\beta}^{\ell}(v) \\
= & \frac{(i)^{q} 2^{\alpha+\beta} \pi^{n}}{(2 \pi)^{n / 2} K_{n}(\alpha) H_{n}(\beta)} \cdot \frac{\Gamma(\alpha / 2) \Gamma(\beta / 2)}{\Gamma((n-\alpha) / 2) \Gamma((n-\beta) / 2)} \\
& \times\left(\sqrt{\sum_{i=1}^{p} \xi_{i}^{2}-\sum_{j=p+1}^{p+q} \xi_{j}^{2}}\right)^{-\alpha}\left(\sqrt{\sum_{i=1}^{n} \xi_{i}^{2}}\right)^{-\beta}, \quad i=\sqrt{-1}
\end{aligned}
$$


In particular, if $\alpha=\beta=2 k, k$ is a nonnegative integer,

$$
(-1)^{k} \widehat{K_{2 k, 2 k}(\xi)}=\frac{1}{(2 \pi)^{n / 2}} \frac{1}{\left(\left(\xi_{1}^{2}+\xi_{2}^{2}+\cdots+\xi_{p}^{2}\right)^{2}-\left(\xi_{p+1}^{2}+\xi_{p+2}^{2}+\cdots+\xi_{p+q}^{2}\right)^{2}\right)^{k}},
$$

where $R_{\alpha}^{H}(u)$ and $R_{\beta}^{\ell}(v)$ are defined by (2.1) and (2.3), respectively.

Proof. See [2, page 194] and [5, pages 156-157].

DefinITION 2.6. The spectrum of the distributional kernel $K_{\alpha, \beta}(x)$ is the support of the Fourier transform $\widehat{K_{\alpha, \beta}(\xi)}$ or the spectrum of $K_{\alpha, \beta}(x)=\operatorname{supp} \widehat{K_{\alpha, \beta}(\xi)}$. Now, from Lemma 2.5 we obtain

$$
\operatorname{supp} \widehat{K_{\alpha, \beta}(\xi)}=\left(\operatorname{supp} \mathfrak{J} R_{\alpha}^{H}(u)\right) \cap\left(\operatorname{supp} \mathfrak{T} R_{\beta}^{\ell}(v)\right) .
$$

In particular, from (2.9) the spectrum of

$$
(-1)^{k} K_{2 k, 2 k}(x)=\operatorname{supp}\left[\frac{1}{(2 \pi)^{n / 2}\left(\left(\sum_{i=1}^{p} \xi_{i}^{2}\right)^{2}-\left(\sum_{j=p+1}^{p+q} \xi_{j}^{2}\right)^{2}\right)^{k}}\right] .
$$

LEMMA 2.7. Let $P\left(x_{1}, x_{2}, \ldots, x_{n}\right)$ be a quadratic form of positive definite, and is defined by

$$
P=P\left(x_{1}, x_{2}, \ldots, x_{n}\right)=\left(\sum_{i=1}^{p} x_{i}^{2}\right)^{2}-\left(\sum_{j=p+1}^{p+q} x_{j}^{2}\right)^{2},
$$

then for any testing function $\varphi(x) \in D$, the space of infinitely differentiable function with compact support,

$$
\begin{aligned}
& \left\langle\delta^{(k)}(P), \varphi\right\rangle=\int_{0}^{\infty}\left[\left(\frac{\partial}{4 s^{3} \partial s}\right)^{k}\left(s^{q-4} \frac{\psi(r, s)}{4}\right)\right]_{s=r} r^{p-1} d r \\
& \left\langle\delta^{(k)}(P), \varphi\right\rangle=(-1)^{k} \int_{0}^{\infty}\left[\left(\frac{\partial}{4 r^{3} \partial r}\right)^{k}\left(r^{p-4} \frac{\psi(r, s)}{4}\right)\right]_{r=s} s^{q-1} d s,
\end{aligned}
$$

where $r^{2}=x_{1}^{2}+x_{2}^{2}+\cdots+x_{p}^{2}, s^{2}=x_{p+1}^{2}+x_{p+2}^{2}+\cdots+x_{p+q}^{2}$, and

$$
\psi(r, s)=\int \varphi d \Omega^{p} d \Omega^{q},
$$

where $d \Omega^{p}$ and $d \Omega^{q}$ are the elements of surface area on the unit sphere in $\mathbb{R}^{p}$ and $\mathbb{R}^{q}$, respectively. Both integrals (2.13) and (2.14) converge if $k<(1 / 4)(p+q-4)$ for any $\varphi(x) \in D$. If $k \geq(1 / 4)(p+q-4)$, these integrals must be understood in the sense of their regularization and (2.13) defined as $\left\langle\delta_{1}^{(k)}(p), \varphi\right\rangle$ and (2.14) defined as $\left\langle\delta_{2}^{(k)}(p), \varphi\right\rangle$. Moreover, if we put $u=r^{2}, v=s^{2}$, thus (2.13) and (2.14) become

$$
\begin{aligned}
& \left\langle\delta^{(k)}(p), \varphi\right\rangle=\frac{1}{16} \int_{0}^{\infty}\left[\frac{\partial^{k}}{\partial v^{k}}\left(v^{(q-4) / 4} \psi_{1}(u, v)\right)\right]_{v=u} u^{(1 / 4)(p-4)} d u, \\
& \left\langle\delta^{(k)}(p), \varphi\right\rangle=\frac{(-1)^{k}}{16} \int_{0}^{\infty}\left[\frac{\partial^{k}}{\partial u^{k}}\left(u^{(p-4) / 4} \psi_{1}(u, v)\right)\right]_{u=v} v^{(1 / 4)(q-4)} d v,
\end{aligned}
$$

where $\psi_{1}(u, v)=\psi(r, s)$. 
Proof. See [2, pages 247-251].

LEMMA 2.8. Let $G_{b}=\left\{\xi \in \mathbb{R}^{n}:\left|\xi_{1}\right| \leq b_{1},\left|\xi_{2}\right| \leq b_{2}, \ldots,\left|\xi_{n}\right| \leq b_{n}\right\}$ be a parallelepiped in $\mathbb{R}^{n}$ and $b_{i}(1 \leq i \leq n)$ is a real constant and the inverse Fourier transform of $\widehat{K_{\alpha, \beta}(\xi)}$ is defined by

$$
K_{\alpha, \beta}(x)=\mathfrak{I}^{-1} \widehat{K_{\alpha, \beta}(\xi)}=\frac{1}{(2 \pi)^{n / 2}} \int_{G_{b}} e^{i(\xi, x)} \widehat{K_{\alpha, \beta}(\xi)} d \xi,
$$

where $K_{\alpha, \beta}$ is defined by (1.4) and $x, \xi \in \mathbb{R}^{n}$, then $K_{\alpha, \beta}(x)$ can be extended to the entire function $K_{\alpha, \beta}(z)$ and be analytic for all $z=\left(z_{1}, z_{2}, \ldots, z_{n}\right) \in \mathbb{C}^{n}$, where $\mathbb{C}^{n}$ is the n-tuple space of complex number and

$$
\left|K_{\alpha, \beta}(z)\right| \leq C \exp (b|\operatorname{Im}(z)|)
$$

where $\exp (b|\operatorname{Im}(z)|)=\exp \left[b_{1}\left|\operatorname{Im}\left(z_{1}\right)\right|+b_{2}\left|\operatorname{Im}\left(z_{2}\right)\right|+\cdots+b_{n}\left|\operatorname{Im}\left(z_{n}\right)\right|\right]$ and $C=$ $\left(1 /(2 \pi)^{n / 2}\right) \int_{G_{b}}\left|\widehat{K_{\alpha, \beta}(\xi)}\right| d \xi$ is a constant. Moreover, $K_{\alpha, \beta}(x)$ has a spectrum contained in $G_{b}$.

Proof. Since the integral of (2.18) converges for all $\xi \in G_{b}$, thus $K_{\alpha, \beta}(x)$ can be extended to the entire function $K_{\alpha, \beta}(z)$ and be analytic for all $z \in C^{n}$. Thus (2.18) can be written as

$$
K_{\alpha, \beta}(z)=\frac{1}{(2 \pi)^{n / 2}} \int_{G_{b}} e^{i(\xi, z)} \widehat{K_{\alpha, \beta}(\xi)} d \xi
$$

Now,

$$
\begin{array}{r}
\left|K_{\alpha, \beta}(z)\right| \leq \frac{1}{(2 \pi)^{n / 2}} \int_{G_{b}}\left|\widehat{K_{\alpha, \beta}(\xi)}\right|\left|\exp \left(i \xi_{1} z_{1}+i \xi_{2} z_{2}+\cdots+i \xi_{n} z_{n}\right)\right| d \xi \\
=\frac{1}{(2 \pi)^{n / 2}} \int_{G_{b}}\left|\widehat{K_{\alpha, \beta}(\xi)}\right| \mid \exp \left(i \xi_{1} \sigma_{1}+i \xi_{2} \sigma_{2}+\cdots+i \xi_{n} \sigma_{n}\right. \\
\left.-\xi_{1} \mu_{1}-\xi_{2} \mu_{2}-\cdots-\xi_{n} \mu_{n}\right) \mid d \xi
\end{array}
$$

where

$$
z_{j}=\sigma+i \mu_{j} \quad(j=1,2, \ldots, n)
$$

thus

$$
\left|K_{\alpha, \beta}(z)\right| \leq \frac{1}{(2 \pi)^{n / 2}} \int_{G_{b}}\left|\widehat{K_{\alpha, \beta}(\xi)}\right| d \xi \exp \left(b_{1}\left|\mu_{1}\right|+b_{2}\left|\mu_{2}\right|+\cdots+b_{n}\left|\mu_{n}\right|\right)
$$

for $\left|\xi_{j}\right| \leq b_{j}$, or $\left|K_{\alpha, \beta}(z)\right| \leq C \exp \left(b_{1}\left|\operatorname{Im}\left(z_{1}\right)\right|+b_{2}\left|\operatorname{Im}\left(z_{2}\right)\right|+\cdots+b_{n}\left|\operatorname{Im}\left(z_{n}\right)\right|\right)$, or $\left|K_{\alpha, \beta}(z)\right| \leq C \exp (b|\operatorname{Im}(z)|)$, where $\left.C=\left(1 /(2 \pi)^{n / 2}\right) \int_{G_{b}} \mid \widehat{K_{\alpha, \beta}(\xi}\right) \mid d \xi$ is a constant.

We must show that the support of $\widehat{K_{\alpha, \beta}(\xi)}$ is contained in $G_{b}$. Since $K_{\alpha, \beta}(z)$ is an analytic function that satisfies the inequality (2.19) and is called an entire function of order of growth $\leq 1$ and of type $\leq b$, then by Paley-Wiener-Schartz theorem, see [3, page 162], $\widehat{K_{\alpha, \beta}(\xi)}$ has a support contained in $G_{b}$, that is the spectrum of $K_{\alpha, \beta}(x)$ is contained in $G_{b}$. 
In particular, for $\alpha=\beta=2 k$, the spectrum of $(-1)^{k} K_{2 k, 2 k}(x)$ is also contained in $G_{b}$, that is $\operatorname{supp}\left[(-1)^{k} \widehat{K_{2 k, 2 k}(\xi)}\right] \subset G_{b}$, where $(-1)^{k} K_{2 k, 2 k}(x)$ is an elementary solution of the Diamond operator $\diamond^{k}$ by Lemma 2.4, and the Fourier transform $(-1)^{k} \widehat{K_{2 k, 2 k}(\xi)}$ given by (2.9) can be defined as follows.

DEFINITION 2.9. The Fourier transform

$$
(-1)^{k} \widehat{K_{2 k, 2 k}(\xi)}= \begin{cases}\frac{1}{(2 \pi)^{n / 2}\left[\left(\sum_{i=1}^{p} \xi_{i}^{2}\right)^{2}-\left(\sum_{j=p+1}^{p+q} \xi_{j}^{2}\right)^{2}\right]^{k}}, & \text { for } \xi \in G_{b}, \\ 0, & \text { for } \xi \in C G_{b},\end{cases}
$$

where $\xi=\left(\xi_{1}, \xi_{2}, \ldots, \xi_{n}\right) \in \mathbb{R}^{n}$ and $C G_{b}$ is the complement of $G_{b}$.

\section{Main results}

THEOREM 3.1. For any nonzero point $\xi \in M$ where $M$ is a spectrum of $(-1)^{k} K_{2 k, 2 k}(x)$, and $(-1)^{k} K_{2 k, 2 k}(x)$ is an elementary solution of the operator $\diamond^{k}$ by Lemma 2.4. Then there exists the residue of the Fourier transform $(-1)^{k} \widehat{K_{2 k, 2 k}(\xi)}$ at the singular point $\lambda=-k$ and such a residue is

$$
\frac{(-1)^{k-1}}{(2 \pi)^{n / 2}(k-1) !} \delta_{1}^{(k-1)(p)} \quad \text { or } \quad \operatorname{res}_{\lambda=-k}(-1)^{k} \widehat{K_{2 k, 2 k}(\xi)}=\frac{(-1)^{k-1}}{(2 \pi)^{n / 2}(k-1) !} \delta^{(k-1)(p)}
$$

where

$$
P=\left(\xi_{1}^{2}+\xi_{2}^{2}+\cdots+\xi_{p}^{2}\right)^{2}-\left(\xi_{p+1}^{2}+\xi_{p+2}^{2}+\cdots+\xi_{p+q}^{2}\right),
$$

$p+q=n$ and $\delta_{1}^{(k-1)}(P)$ is defined by (2.16) with $\delta^{(k-1)}(P)=\delta_{1}^{(k-1)}(P)$ and $n$ is odd with $p$ odd, $q$ even.

Proof. We define the generalized function $P^{\lambda}$, where $P$ is given by (3.2) and $\lambda$ is a complex number, by

$$
\left\langle P^{\lambda}, \varphi\right\rangle=\int_{P>0} P^{\lambda}(\xi) \varphi(\xi) d \xi,
$$

where $\xi=\left(\xi_{1}, \xi_{2}, \ldots, \xi_{n}\right)$ and $d \xi=d \xi_{1} d \xi_{2} \cdots d \xi_{n}$ and $\varphi(\xi) \in D$, the space of continuous infinitely differentiable function with compact support. Now,

$$
\left\langle P^{\lambda}, \varphi\right\rangle=\int_{P>0}\left[\left(\xi_{1}^{2}+\xi_{2}^{2}+\cdots+\xi_{p}^{2}\right)^{2}-\left(\xi_{p+1}^{2}+\xi_{p+2}^{2}+\cdots+\xi_{p+q}^{2}\right)\right]^{\lambda} \varphi(\xi) d \xi .
$$

We transform to bipolar coordinates defined by

$$
\begin{gathered}
\xi_{1}=r w_{1}, \xi_{2}=r w_{2}, \ldots, \xi_{p}=r w_{p}, \\
\xi_{p+1}=s w_{p+1}, \xi_{p+2}=s w_{p+2}, \ldots, \xi_{p+q}=s w_{p+q}, \quad p+q=n,
\end{gathered}
$$

where $\sum_{i=1}^{p} w_{i}^{2}=1$ and $\sum_{j=p+1}^{p+q} w_{j}^{2}=1$. Thus

$$
r=\sqrt{\sum_{i=1}^{p} \xi_{i}^{2}}, \quad s=\sqrt{\sum_{j=p+1}^{p+q} \xi_{j}^{2}}
$$


We have $\left\langle P^{\lambda}, \varphi\right\rangle=\int\left[r^{4}-s^{4}\right]^{\lambda} \varphi(\xi) d \xi$. Since the volume $d \xi=r^{p-1} s^{q-1} d r d s d \Omega_{p} d \Omega_{q}$ where $d \Omega_{p}$ and $d \Omega_{q}$ are the elements of surface area on the unit sphere in $\mathbb{R}^{p}$ and $\mathbb{R}^{q}$, respectively. Thus

$$
\begin{aligned}
\left\langle P^{\lambda}, \varphi\right\rangle & =\int_{p>0}\left(r^{4}-s^{4}\right)^{\lambda} \varphi r^{p-1} s^{q-1} d r d s d \Omega^{p} d \Omega^{q} \\
& =\int_{0}^{\infty} \int_{0}^{r}\left(r^{4}-s^{4}\right)^{\lambda} \psi(r, s) r^{p-1} s^{q-1} d s d r,
\end{aligned}
$$

where $\psi(r, s)=\int \varphi d \Omega_{p} d \Omega_{q}$.

Since $\varphi(\xi)$ is in $D$, then $\psi(r, s)$ is an infinitely differentiable function of $r^{4}$ and $s^{4}$ with bounded support. We now make the change of variable $u=r^{4}, v=s^{4}$, and writing $\psi(r, s)=\psi_{1}(u, v)$. Thus we obtain

$$
\left\langle P^{\lambda}, \varphi\right\rangle=\frac{1}{16} \int_{u=0}^{\infty} \int_{v=0}^{u}(u-v)^{\lambda} \psi_{1}(u, v) u^{(p-4) / 4} v^{(q-4) / 4} d v d u
$$

Write $v=u t$. We obtain

$$
\left\langle P^{\lambda}, \varphi\right\rangle=\frac{1}{16} \int_{0}^{\infty} u^{\lambda+(1 / 4)(p+q)-1} d u \int_{0}^{1}(1-t)^{\lambda} t^{(q-4) / 4} \psi_{1}(u, u t) d t .
$$

Let the function

$$
\Phi(\lambda, u)=\frac{1}{16} \int_{0}^{1}(1-t)^{\lambda} t^{(q-4) / 4} \psi_{1}(u, u t) d t .
$$

Thus $\Phi(\lambda, u)$ has singularity at $\lambda=-k$ where it has simple poles. By Gel'fand and Shilov [2, page 254, equation (12)] we obtain the residue of $\Phi(\lambda, u)$ at $\lambda=-k$, that is,

$$
\operatorname{res}_{\lambda=-k} \Phi(\lambda, u)=\frac{1}{16} \frac{(-1)^{k-1}}{(k-1) !}\left[\frac{\partial^{k-1}}{\partial t^{k-1}}\left\{t^{(q-4) / 4} \psi_{1}(u, u t)\right\}\right]_{t=1} .
$$

Thus, $\operatorname{res}_{\lambda=-k} \Phi(\lambda, u)$ is a functional concentrated on the surface $P=0(t=1, u=v$, $p=u-v=0$ ). On the other hand, from (3.9) and (3.10) we have

$$
\left\langle P^{\lambda}, \varphi\right\rangle=\int_{0}^{\infty} u^{\lambda+(1 / 4)(p+q)-1} \Phi(\lambda, u) d u .
$$

Thus $\left\langle P^{\lambda}, \varphi\right\rangle$ in (3.12) has singularities at $\lambda=-n / 4,-n / 4-1, \ldots,-n / 4-k$. At these points,

$$
\operatorname{res}_{\lambda=-n / 4-k}\left\langle P^{\lambda}, \varphi\right\rangle=\frac{1}{k !}\left[\frac{\partial^{k}}{\partial u^{k}} \Phi\left(-\frac{n}{4}-k, u\right)\right]_{u=0} .
$$

Thus the residue of $\left\langle P^{\lambda}, \varphi\right\rangle$ at $\lambda=(-1 / 2) n-k$ is a functional concentrated on the vertex of the surface $P$. Now consider the case when the singular point $\lambda=-k$. Write (3.10) in the neighborhood of $\lambda=-k$ in the form $\Phi(\lambda, u)=\Phi_{0}(u) /(\lambda+k)+\Phi_{1}(\lambda, u)$ where $\Phi_{0}(u)=\operatorname{res}_{\lambda=-k} \Phi(\lambda, u)$ and $\Phi_{1}(\lambda, u)$ is regular at $\lambda=-k$. Substitute $\Phi(\lambda, u)$ into (3.12) we obtain

$$
\left\langle P^{\lambda}, \varphi\right\rangle=\frac{1}{\lambda+k} \int_{0}^{\infty} u^{\lambda+(1 / 4)(p+q)-1} \Phi_{0}(u) d u+\int_{0}^{\infty} u^{\lambda+(1 / 4)(p+q)-1} \Phi_{1}(\lambda, u) d u .
$$


Thus $\operatorname{res}_{\lambda=-k}\left\langle P^{\lambda}, \varphi\right\rangle=\int_{0}^{\infty} u^{-k+(1 / 4)(p+q)-1} \Phi_{0}(u) d u$. By substituting $\Phi_{0}(u)$ and (3.11), we obtain

$$
\operatorname{res}_{\lambda=-k}\left\langle P^{\lambda}, \varphi\right\rangle=\frac{(-1)^{k}}{16(k-1) !} \int_{0}^{\infty}\left[\frac{\partial^{k-1}}{\partial t^{k-1}}\left\{t^{1(q-4) / 4} \psi_{1}(u, u t)\right\}\right]_{t=1} u^{-k+(1 / 4)(p+q)-1} d u
$$

since, we put $v=u t$. Thus $\partial^{k-1} / \partial t^{k-1}=u^{k-1}\left(\partial^{k-1} / \partial v^{k-1}\right)$, by substituting $\partial^{k-1} / \partial t^{k-1}$ we obtain

$$
\operatorname{res}_{\lambda=-k}\left\langle P^{\lambda}, \varphi\right\rangle=\frac{(-1)^{k}}{16(k-1) !} \int_{0}^{\infty}\left[\frac{\partial^{k-1}}{\partial t^{k-1}}\left\{v^{1(q-4) / 4} \psi_{1}(u, v)\right\}\right]_{u=v} u^{(1 / 4) p-1} d u .
$$

Now, by (2.16)

$$
\operatorname{res}_{\lambda=-k}\left\langle P^{\lambda}, \varphi\right\rangle=\frac{(-1)^{k-1}}{(k-1) !} \delta_{1}^{(k-1)}(P) .
$$

Since, by Definition 2.9 we have

$$
(-1)^{k} \widehat{K_{2 k, 2 k}(\xi)}=\frac{1}{(2 \pi)^{n / 2}} P^{\lambda} \text { for } \lambda=-k,
$$

and $\xi \in G_{b}$. Let $M$ be a spectrum of $(-1)^{k} K_{2 k, 2 k}(x)$ and $M \subset G_{b}$ by Lemma 2.8. Thus for any nonzero $\xi \in M$ we can find the residue of $(-1)^{k} \widehat{K_{2 k, 2 k}(\xi)}$, that is,

$$
\begin{aligned}
\operatorname{res}_{\lambda=-k}\left\langle(-1)^{k} \widehat{K_{2 k, 2 k}(\xi)}, \varphi(\xi)\right\rangle & =\frac{1}{(2 \pi)^{n / 2}} \operatorname{res}_{\lambda=-k}\left\langle P^{\lambda}, \varphi\right\rangle \\
& =\frac{(-1)^{k-1}}{(2 \pi)^{n / 2}(k-1) !}\left\langle\delta_{1}^{(k-1)}(P), \varphi\right\rangle
\end{aligned}
$$

or $\operatorname{res}_{\lambda=-k}(-1)^{k} \widehat{K_{2 k, 2 k}(\xi)}=\left((-1)^{k-1} /\left(2(\pi)^{n / 2}(k-1) !\right)\right) \delta_{1}^{(k-1)}(P)$ for $\xi \in M$ and $\xi \neq 0$.

Now consider the case $\xi=0$. We have from (3.13) that, the residue of $\left\langle P^{\lambda}, \varphi\right\rangle$ occurs at the point $\lambda=(-1 / 2) n-k$ that is $\operatorname{res}_{\lambda=-(1 / 2) n-k}\left\langle P^{\lambda}, \varphi\right\rangle$ is a functional concentrated on the vertex of surface $P$. Since $u=0$ and $v=u t$, then $u=v=0$, that implies

$$
\sqrt{\xi_{1}^{2}+\xi_{2}^{2}+\cdots+\xi_{p}^{2}}=\sqrt{\xi_{p+1}^{2}+\xi_{p+2}^{2}+\cdots+\xi_{p+q}^{2}}=0 .
$$

It follows that $\xi_{1}=\xi_{2}=\cdots=\xi_{p+q}=0, p+q=n$. Thus, the residue of $\left\langle P^{\lambda}, \varphi\right\rangle$ is concentrated on the point $\xi=0$.

Since, from Definition 2.9, $\left(1 /(2 \pi)^{n / 2}\right) P^{\lambda}=(-1)^{k} \widehat{K_{2 k, 2 k}(\xi)}$ if $\lambda=-k$. Thus we only consider the residue of $(-1)^{k} \widehat{K_{2 k, 2 k}(\xi)}$ at $\lambda=-k$. From (3.12), we consider the residue of $\left\langle P^{\lambda}, \varphi\right\rangle$ only at $\lambda=-k$. That implies $(1 / 4)(p+q)-1=0$ or $n=4(p+q=n)$. Since $n=4$ is an even dimension which contradicts Lemma 2.4, the existence of the elementary solution $(-1)^{k} K_{2 k, 2 k}(x)$ that exists for odd $n$. Thus cases (3.12) and (3.13) do not occur. This implies that the case $\xi=0$ does not happen. It follows that

$$
\operatorname{res}_{\lambda=-k}(-1)^{k} \widehat{K_{2 k, 2 k}(\xi)}=\frac{(-1)^{k-1}}{(2 \pi)^{n / 2}(k-1) !} \delta_{1}^{(k-1)}(P)
$$

for nonzero point $\xi \in M$ concentrated on the surface $P=0$, where $M$ is a spectrum of $(-1)^{k} K_{2 k, 2 k}(x)$. 
ACKNOWLEDGEMENTS. The author would like to thank the Thailand Research Fund for financial support and particularly Prof Dr Virulh Sa-yakanit who gave some partial supports.

\section{REFERENCES}

[1] M. A. Aguirre Téllez, The distributional Hankel transform of Marcel Riesz's ultrahyperbolic kernel, Stud. Appl. Math. 93 (1994), no. 2, 133-162. MR 95k:46066. Zbl 820.46037.

[2] I. M. Gel'fand and G. E. Shilov, Generalized Functions. Vol. 1. Properties and Operations, Academic Press, New York, 1964. MR 55\#8786a. Zbl 115.33101.

[3] _ Generalized Functions. Vol. 2. Spaces of Fundamental and Generalized Functions, Academic Press, New York, 1968. MR 55\#8786b. Zbl 159.18301.

[4] A. Kananthai, On the solutions of the n-dimensional diamond operator, Appl. Math. Comput. 88 (1997), no. 1, 27-37. MR 98i:35133. Zbl 922.47042.

[5] _ On the Fourier transform of the diamond kernel of Marcel Riesz, Appl. Math. Comput. 101 (1999), no. 2-3, 151-158. MR 2000a:46061. Zbl 939.46023.

[6] Y. Nozaki, On Riemann-Liouville integral of ultra-hyperbolic type, Kōdai Math. Sem. Rep. 16 (1964), 69-87. MR 29\#4906. Zbl 168.37201.

[7] S. E. Trione, On Marcel Riesz's ultra-hyperbolic kernel, Trabajos de Matematica 116 (1987), $1-12$.

[8] A. H. Zemanian, Distribution Theory and Transform Analysis. An Introduction to Generalized Functions, with Applications, McGraw-Hill, New York, 1965. MR 31\#1556. Zbl 127.07201.

Amnuay Kananthai: Department of Mathematics, Chiangmai University, Chiangmai 50200, THAILAND

E-mail address: ma1amnka@science.cmu . ac.th 


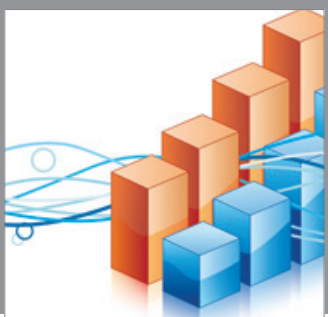

Advances in

Operations Research

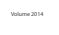

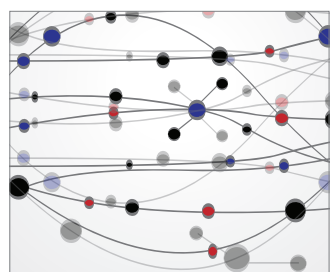

\section{The Scientific} World Journal
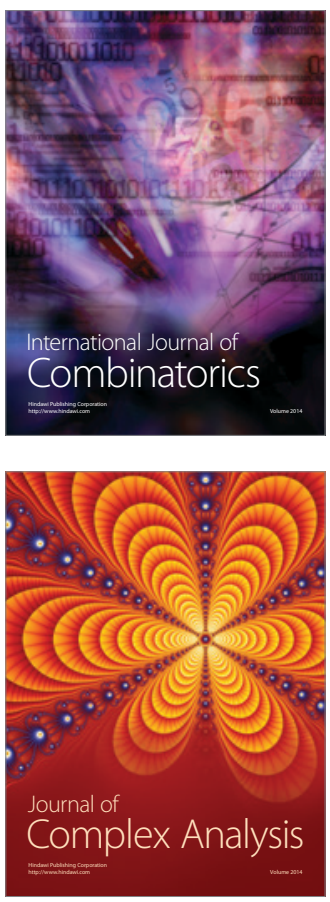

International Journal of

Mathematics and

Mathematical

Sciences
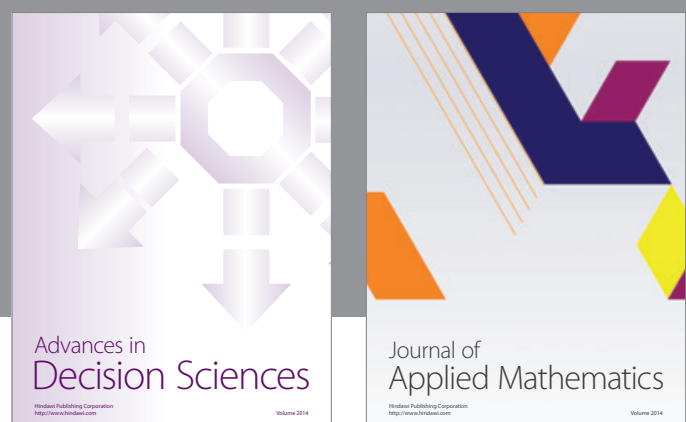

Journal of

Applied Mathematics
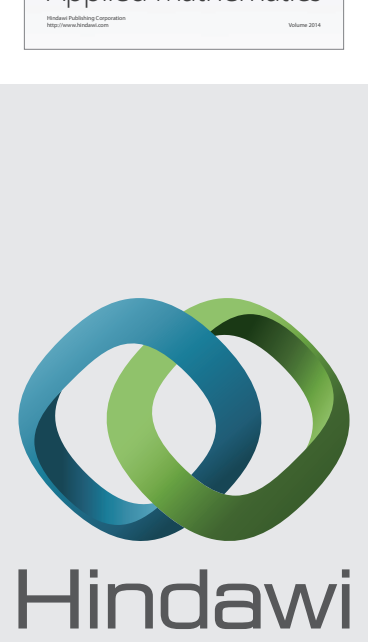

Submit your manuscripts at http://www.hindawi.com
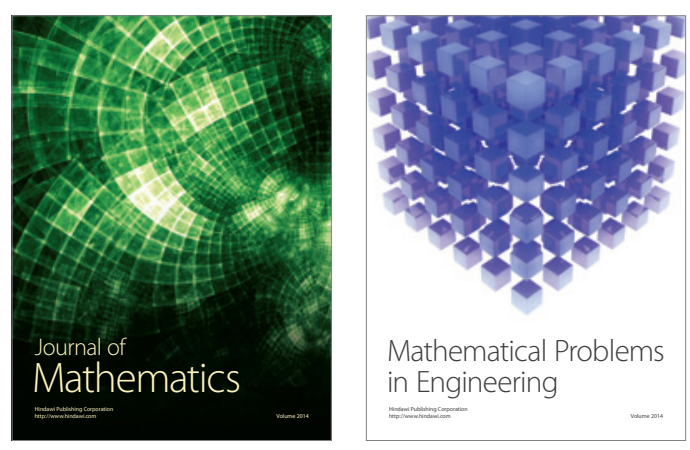

Mathematical Problems in Engineering
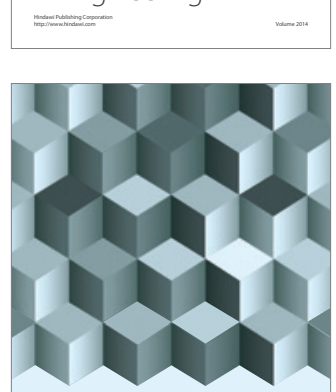

Journal of

Function Spaces
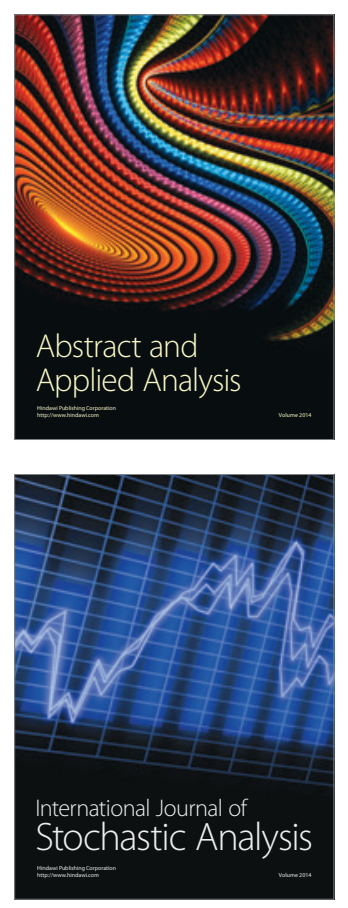

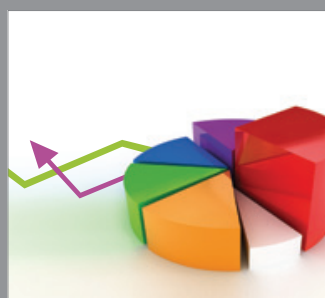

ournal of

Probability and Statistics

Promensencen
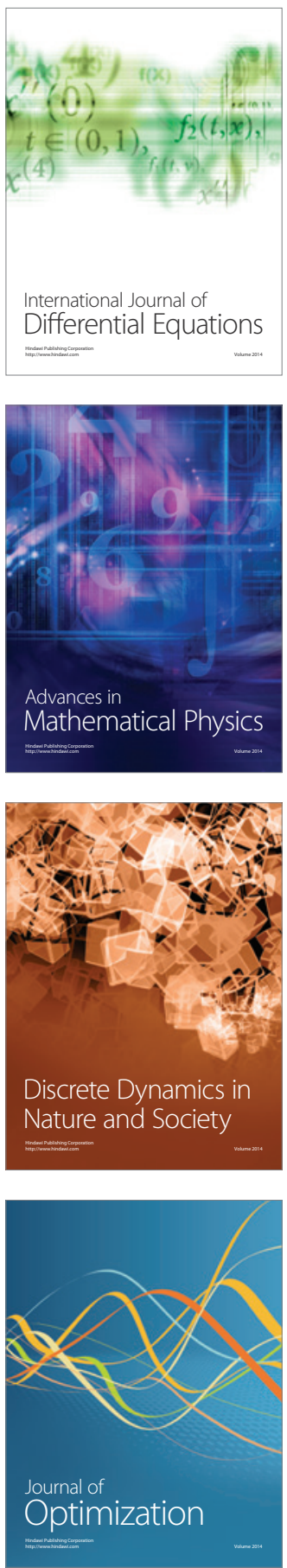